\title{
DYNAMIC SIGNATURES OF COHERENT SYSTEMS BASED ON SEQUENTIAL ORDER STATISTICS
}

\author{
M. BURKSCHAT, ${ }^{*}$ Otto-von-Guericke University Magdeburg \\ J. NAVARRO, ${ }^{* *}$ Universidad de Murcia
}

\begin{abstract}
Sequential order statistics can be used to describe the ordered lifetimes of components in a system, where the failure of a component may affect the performance of remaining components. In this paper mixture representations of the residual lifetime and the inactivity time of systems with such failure-dependent components are considered. Stochastic comparisons of differently structured systems are obtained and properties of the weights in the mixture representations are examined. Furthermore, corresponding representations of the residual lifetime and the inactivity time of a system given the additional information about a previous failure time are derived.
\end{abstract}

Keywords: Signature; coherent system; sequential order statistics; mixture; mixed system

2010 Mathematics Subject Classification: Primary 62E15

Secondary $60 \mathrm{~K} 10$

\section{Introduction}

For assessing the reliability of technical systems that consist of several components, it is of interest to study probability models that describe the system lifetime by taking into account the component lifetimes. The lifetime $T$ of a coherent system can be represented as $T=$ $\phi\left(X_{1}, \ldots, X_{n}\right)$, where $\phi$ denotes a structure function and $X_{1}, \ldots, X_{n}$ denote random variables that describe the component lifetimes (see Barlow and Proschan $(1981$, p. 12)). Under the usual assumption of independent and identically distributed (i.i.d.) component lifetimes, it can be shown that

$$
\mathbb{P}(T>t)=\sum_{i=1}^{n} s_{i} \mathbb{P}\left(X_{i: n}>t\right), \quad t \in \mathbb{R},
$$

where $s_{1}, \ldots, s_{n}$ denote appropriately chosen nonnegative coefficients and $X_{1: n} \leq \cdots \leq X_{n: n}$ are the order statistics of the component lifetimes $X_{1}, \ldots, X_{n}$. For i.i.d. component lifetimes with a common continuous distribution function, the preceding representation has been obtained in Samaniego (1985). The vector $s=\left(s_{1}, \ldots, s_{n}\right)$ is called the system signature. Under these assumptions, the $i$ th entry of the signature vector satisfies $s_{i}=\mathbb{P}\left(T=X_{i: n}\right)$, that is, it coincides with the probability that the $i$ th failure of a component leads to the system failure. For a survey of applications of system signatures, we refer the reader to Samaniego (2007).

The i.i.d. assumption in (1.1) can in fact be relaxed. Navarro and Rychlik (2007) have shown that representation (1.1) is also valid for exchangeable random variables $X_{1}, \ldots, X_{n}$ with an

Received 3 January 2012; revision received 11 June 2012.

* Postal address: Institute of Mathematical Stochastics, Otto-von-Guericke University Magdeburg, D-39016 Magdeburg, Germany. Email address: marco.burkschat@ovgu.de

** Postal address: Facultad de Matemáticas, Universidad de Murcia, 30100 Murcia, Spain.

Email address: jorgenav@um.es 
absolutely continuous joint distribution. Moreover, Navarro et al. (2008b) proved that (1.1) still holds for arbitrary exchangeable random variables $X_{1}, \ldots, X_{n}$, if the signature vector is obtained by considering a system with the same structure and i.i.d. component lifetimes from a common continuous distribution instead of the original system. In all these cases, the signature is given by the ratio of the number of orderings for which the $i$ th failure of a component causes the system failure to the total number, $n$ !, of possible orderings of the $n$ component lifetimes (cf. Samaniego (2007, p. 23)). In particular, the system signature depends only on the structure function and the number of components in the system.

In general, if a component in a technical system fails, the performance of the remaining components might be affected by the failure. In order to take into account such phenomena, Kamps (1995a) (see also Kamps (1995b)) introduced the model of sequential order statistics. These random variables describe the increasingly ordered failure times of components in a sequential $k$-out-of- $n$ system (see Cramer and Kamps (2001)).

Sequential order statistics can be motivated as follows. In the following, let $F_{1}, \ldots, F_{n}$ denote continuous distribution functions with support $[0, \infty)$. Let us assume that at time $t=0$ all the $n$ components in a system start to work. The lifetimes of the components are described by independent random variables $X_{1}^{(1)}, \ldots, X_{n}^{(1)}$ which are identically distributed according to the distribution function $F_{1}$. The first failure time of a component is then given by

$$
X_{1: n}^{*}=\min \left\{X_{1}^{(1)}, \ldots, X_{n}^{(1)}\right\} .
$$

If this first failure occurs at time $t_{1}=X_{1: n}^{*}$ then we assume that the residual lifetimes of the $n-1$ remaining components are equal in law to the residual lifetimes of i.i.d. components with age $t_{1}$ and distribution function $F_{2}$ (instead of $F_{1}$ ). Here the distribution function $F_{2}$ is used to model the influence of the first component failure on the behavior of the other components. The lifetimes of such components will be represented by random variables $X_{1}^{(2)}, \ldots, X_{n-1}^{(2)}$. Given that $X_{1: n}^{*}=t_{1}$, the random variables $X_{1}^{(2)}, \ldots, X_{n-1}^{(2)}$ are i.i.d. according to the truncated distribution function $F_{2}\left(\cdot \mid t_{1}\right)$ with $\bar{F}_{2}\left(x \mid t_{1}\right)=\bar{F}_{2}(x) / \bar{F}_{2}\left(t_{1}\right)$ for $x \geq t_{1}$. In particular, given that $X_{1: n}^{*}=t_{1}$, these random variables satisfy $X_{i}^{(2)} \geq t_{1}$ for $i=1, \ldots, n-1$. Then, the second failure time in the system is given by

$$
X_{2: n}^{*}=\min \left\{X_{1}^{(2)}, \ldots, X_{n-1}^{(2)}\right\} .
$$

We proceed this way and, by induction, we assume that the $i$ th component failure occurs at time $t_{i}=X_{i: n}^{*}(1 \leq i \leq n-1)$. Then we assume that the residual lifetimes of the $n-i$ remaining components are equal in law to the residual lifetimes of i.i.d. components with age $t_{i}$ and distribution function $F_{i+1}$. The lifetimes of such components will be represented by $X_{1}^{(i+1)}, \ldots$, $X_{n-i}^{(i+1)}$. Given that $X_{i: n}^{*}=t_{i}$, the random variables $X_{1}^{(i+1)}, \ldots, X_{n-i}^{(i+1)}$ are i.i.d. according to the truncated distribution function $F_{i+1}\left(\cdot \mid t_{i}\right)$ with $\bar{F}_{i+1}\left(x \mid t_{i}\right)=\bar{F}_{i+1}(x) / \bar{F}_{i+1}\left(t_{i}\right)$ for $x \geq t_{i}$. Then, the $(i+1)$ th component failure time is

$$
X_{i+1: n}^{*}=\min \left\{X_{1}^{(i+1)}, \ldots, X_{n-i}^{(i+1)}\right\} .
$$

In particular, $X_{n: n}^{*}$ denotes the failure time of the last component in the system. The resulting ordered lifetimes $X_{1: n}^{*} \leq \cdots \leq X_{n: n}^{*}$ are called sequential order statistics based on $F_{1}, \ldots, F_{n}$. The distribution functions $F_{2}, \ldots, F_{n}$ are used to describe the effects of failures on remaining components. If $F_{1}=\cdots=F_{n}$ (i.e. when the failure of a component does not affect the other components) then their joint distribution coincides with the distribution of usual order statistics 
based on $n$ i.i.d. random variables that are distributed according to $F_{1}$. For further details about the definition of sequential order statistics, we refer the reader to Kamps (1995a) (see also Cramer and Kamps (2001), Cramer and Kamps (2003), and Cramer (2006)).

Recently, the idea underlying sequential order statistics has been extended to general coherent systems (see Burkschat (2009) and Navarro and Burkschat (2011)). In order to describe component lifetimes in a system, where failures might change the lifetimes of remaining components, a particular dependence model for the components is assumed. The vector of component lifetimes $\left(X_{1}^{*}, \ldots, X_{n}^{*}\right)$ is chosen to be an exchangeable random vector such that the vector of associated order statistics coincides with sequential order statistics $\left(X_{1: n}^{*}, \ldots, X_{n: n}^{*}\right)$ based on $F_{1}, \ldots, F_{n}$. Then $T=\phi\left(X_{1}^{*}, \ldots, X_{n}^{*}\right)$ describes the lifetime of a coherent system, where failures can affect the lifetimes of remaining components. In the following, such systems are called coherent systems based on sequential order statistics or coherent systems with failuredependent component lifetimes $X_{1}^{*}, \ldots, X_{n}^{*}$ (based on $\left.F_{1}, \ldots, F_{n}\right)$. Furthermore, note that the corresponding system lifetime has the representation

$$
\mathbb{P}(T>t)=\sum_{i=1}^{n} s_{i} \mathbb{P}\left(X_{i: n}^{*}>t\right), \quad t \in \mathbb{R},
$$

where $s=\left(s_{1}, \ldots, s_{n}\right)$ denotes the signature vector. For applications of this representation to orderings of system lifetimes, we refer the reader to Navarro and Burkschat (2011). Related results can also be found in Burkschat and Navarro (2011).

The notion of a coherent system has been extended by introducing the concept of a mixed system (see, e.g. Samaniego (2007, p. 29)). For describing the lifetime of a mixed system, the vector $s=\left(s_{1}, \ldots, s_{n}\right)$ in the mixture representation (1.1) is allowed to be an arbitrary probability vector, that is, the entries only have to satisfy $s_{i} \geq 0$ for $i=1, \ldots, n$ and $s_{1}+\cdots+$ $s_{n}=1$. Such a lifetime can be interpreted as the result of randomly selecting among different coherent systems. By analogy, mixed systems with failure-dependent components are obtained by using arbitrary probability vectors $s$ in the corresponding representation (1.2).

In this paper we study mixture representations of the residual lifetime and the inactivity time of systems with failure-dependent components. Stochastic comparisons of differently structured systems are obtained and properties of the weights in the mixture representations are examined. The results extend known results for systems with i.i.d. component lifetimes given in Navarro et al. (2008a) and Zhang (2010a). Further related results can be found, for instance, in Navarro and Shaked (2006) and Zhang (2010b). Moreover, we derive representations of the residual lifetime and the inactivity time of a system with failure-dependent components given the additional information about a previous failure time.

In this paper we frequently focus on sequential order statistics with underlying distribution functions given by

$$
F_{i}(x)=1-(1-F(x))^{\alpha_{i}}, \quad x \in \mathbb{R},
$$

where $F$ denotes a continuous distribution function and $\alpha_{1}, \ldots, \alpha_{n}>0$. We also assume that the support of $F$ is given by the interval $[0, \infty)$ for simplicity. If $F$ is absolutely continuous with density $f$ then the corresponding hazard rates satisfy

$$
\lambda_{F_{i}}(x)=\alpha_{i} \lambda_{F}(x), \quad i=1, \ldots, n .
$$

Therefore, (1.3) is usually referred as the proportional hazard rate (PHR) assumption. It is well known that in this case sequential order statistics can be treated as generalized order statistics. 
For further information on the relation between sequential and generalized order statistics, we again refer the reader to Kamps (1995a), Cramer and Kamps (2001), (2003), and Cramer (2006) (see also Section 2).

\section{Mixture representations of residual lifetimes and inactivity times}

In the following we consider mixture representations of the residual lifetime and the inactivity time of a system. The first result of Theorem 2.1 below was obtained in Navarro and Burkschat (2011) and extends a result given in Navarro et al. (2008a). The second representation is shown similarly and, therefore, the derivation is omitted. For systems with i.i.d. components, the corresponding result was shown in Zhang (2010a).

Theorem 2.1. Let $\boldsymbol{s}=\left(s_{1}, \ldots, s_{n}\right)$ denote the signature of a mixed system with failuredependent component lifetimes $X_{1}^{*}, \ldots, X_{n}^{*}$ based on $F_{1}, \ldots, F_{n}$, and let $T$ denote the corresponding system lifetime.

(a) Let $x \geq 0$ and $t \geq 0$. Then

$$
\mathbb{P}(T-t>x \mid T>t)=\sum_{i=1}^{n} p_{i}(t) \mathbb{P}\left(X_{i: n}^{*}-t>x \mid X_{i: n}^{*}>t\right)
$$

with

$$
p_{i}(t)=\frac{s_{i} \mathbb{P}\left(X_{i: n}^{*}>t\right)}{\sum_{k=1}^{n} s_{k} \mathbb{P}\left(X_{k: n}^{*}>t\right)}, \quad i=1, \ldots, n .
$$

(b) Let $0 \leq x \leq t$. Then

$$
\mathbb{P}(t-T>x \mid T \leq t)=\sum_{i=1}^{n} \tilde{p}_{i}(t) \mathbb{P}\left(t-X_{i: n}^{*}>x \mid X_{i: n}^{*} \leq t\right)
$$

with

$$
\tilde{p}_{i}(t)=\frac{s_{i} \mathbb{P}\left(X_{i: n}^{*} \leq t\right)}{\sum_{k=1}^{n} s_{k} \mathbb{P}\left(X_{k: n}^{*} \leq t\right)}, \quad i=1, \ldots, n .
$$

In the following, we apply the notation

$$
\boldsymbol{p}(t)=\left(p_{1}(t), \ldots, p_{n}(t)\right), \quad \tilde{\boldsymbol{p}}(t)=\left(\tilde{p}_{1}(t), \ldots, \tilde{p}_{n}(t)\right) .
$$

Several of the subsequent results are shown by imposing the PHR assumption (1.3). Given this assumption, the joint distribution of sequential order statistics coincides with that of generalized order statistics with the model parameters

$$
\gamma_{i}=\alpha_{i}(n-i+1), \quad i=1, \ldots, n
$$

(see, e.g. Cramer and Kamps (2001)). For this reason, it is helpful to briefly recall the definition of generalized order statistics (see Kamps (1995a) or Kamps (1995b)). Uniform generalized order statistics $U_{1: n}^{*}, \ldots, U_{n: n}^{*}$ are introduced by specifying their joint density function as

$$
\begin{aligned}
& f_{U_{1: n}^{*}, \ldots, U_{n: n}^{*}}\left(u_{1}, \ldots, u_{n}\right) \\
& \quad=\left(\prod_{j=1}^{n} \gamma_{j}\right)\left(\prod_{j=1}^{n-1}\left(1-u_{j}\right)^{\gamma_{j}-\gamma_{j+1}-1}\right)\left(1-u_{n}\right)^{\gamma_{n}-1}, \quad 0 \leq u_{1} \leq \cdots \leq u_{n}<1,
\end{aligned}
$$


with the model parameters $\gamma_{1}, \ldots, \gamma_{n}>0$. Generalized order statistics $X_{1: n}^{*}, \ldots, X_{n: n}^{*}$ based on the distribution function $F$ are then obtained via the quantile transformation

$$
X_{r: n}^{*}=F^{-1}\left(U_{r: n}^{*}\right), \quad r=1, \ldots, n .
$$

In the proofs of the next results, we use the fact that generalized order statistics are ordered according to the likelihood ratio ordering, i.e. $X_{r: n}^{*} \leq_{\operatorname{lr}} X_{r+1: n}^{*}$ for $r=1, \ldots, n-1$ (see, for instance, Cramer et al. (2003) or Hu and Zhuang (2005)). Here and in the following, the notation ' $\leq_{\mathrm{st}}$ ', ' $\leq_{\mathrm{hr}}$ ', ' $\leq_{\mathrm{rh}}$ ', and ' $\leq_{\mathrm{lr}}$ ' refer to the stochastic, hazard rate, reversed hazard rate, and likelihood ratio orderings, respectively. Detailed information about these orderings can be found, for instance, in Chapter 1 of Shaked and Shanthikumar (2007).

Theorem 2.2. Let $T_{1}$ and $T_{2}$ denote the lifetimes of two mixed systems with the same failuredependent component lifetimes $X_{1}^{*}, \ldots, X_{n}^{*}$. Let the PHR assumption (1.3) hold.

(a) Let $\boldsymbol{p}_{1}(t)$ and $\boldsymbol{p}_{2}(t)$ denote the vectors of coefficients (2.2) of $T_{1}$ and $T_{2}$, respectively. Then the following statements hold for the residual lifetimes of the systems.

- If $\boldsymbol{p}_{1}(t) \leq_{\mathrm{st}} \boldsymbol{p}_{2}(t)$ then $\left(T_{1}-t \mid T_{1}>t\right) \leq_{\mathrm{st}}\left(T_{2}-t \mid T_{2}>t\right)$.

- If $\boldsymbol{p}_{1}(t) \leq_{\mathrm{hr}} \boldsymbol{p}_{2}(t)$ then $\left(T_{1}-t \mid T_{1}>t\right) \leq_{\mathrm{hr}}\left(T_{2}-t \mid T_{2}>t\right)$.

- If $\boldsymbol{p}_{1}(t) \leq \operatorname{lr} \boldsymbol{p}_{2}(t)$ and $F$ is absolutely continuous, then $\left(T_{1}-t \mid T_{1}>t\right) \leq \mathrm{lr}$ $\left(T_{2}-t \mid T_{2}>t\right)$.

(b) Let $\widetilde{\boldsymbol{p}}_{1}(t)$ and $\widetilde{\boldsymbol{p}}_{2}(t)$ denote the vectors of coefficients (2.4) of $T_{1}$ and $T_{2}$, respectively. Then the following statements hold for the inactivity times of the systems.

- If $\tilde{\boldsymbol{p}}_{1}(t) \leq_{\mathrm{st}} \tilde{\boldsymbol{p}}_{2}(t)$ then $\left(t-T_{1} \mid T_{1} \leq t\right) \geq_{\mathrm{st}}\left(t-T_{2} \mid T_{2} \leq t\right)$.

- If $\tilde{\boldsymbol{p}}_{1}(t) \leq_{\mathrm{rh}} \tilde{\boldsymbol{p}}_{2}(t)$ then $\left(t-T_{1} \mid T_{1} \leq t\right) \geq_{\mathrm{rh}}\left(t-T_{2} \mid T_{2} \leq t\right)$.

- If $\tilde{\boldsymbol{p}}_{1}(t) \leq \operatorname{lr} \widetilde{\boldsymbol{p}}_{2}(t)$ and $F$ is absolutely continuous, then $\left(t-T_{1} \mid T_{1} \leq t\right) \geq_{\mathrm{l}}$ $\left(t-T_{2} \mid T_{2} \leq t\right)$.

Proof. Since $X_{i: n}^{*} \leq \operatorname{lr} X_{i+1: n}^{*}$ for $i=1, \ldots, n-1$, it follows from Theorems 1.C.8 and 1.C.6 of Shaked and Shanthikumar (2007) that

$$
\left(X_{i: n}^{*}-t \mid X_{i: n}^{*}>t\right) \leq_{\mathrm{lr}}\left(X_{i+1: n}^{*}-t \mid X_{i+1: n}^{*}>t\right), \quad i=1, \ldots, n-1 .
$$

Then (a) is obtained by utilizing representation (2.1) and applying the preservation results for mixtures given in Theorems 1.A.6, 1.B.14, and 1.C.17 of Shaked and Shanthikumar (2007). The assertion in (b) follows similarly from (2.3).

Lemma 2.1. Let $\boldsymbol{p}(t)$ and $\widetilde{\boldsymbol{p}}(t)$ denote the respective vectors of coefficients (2.2) and (2.4). Under the PHR assumption (1.3), $\boldsymbol{p}\left(t_{1}\right) \leq_{\operatorname{lr}} \boldsymbol{p}\left(t_{2}\right)$ and $\widetilde{\boldsymbol{p}}\left(t_{1}\right) \leq_{\operatorname{lr}} \widetilde{\boldsymbol{p}}\left(t_{2}\right)$ hold for all $0 \leq t_{1} \leq t_{2}$.

Proof. First we note that $\boldsymbol{p}\left(t_{1}\right)$ and $\boldsymbol{p}\left(t_{2}\right)$ considered as discrete distributions on $\{1, \ldots, n\}$ have the same support. Let us consider $1 \leq i<j \leq n$ in this support. Then

$$
\frac{p_{i}\left(t_{1}\right)}{p_{i}\left(t_{2}\right)}=\frac{s_{i} \mathbb{P}\left(X_{i: n}^{*}>t_{1}\right)}{s_{i} \mathbb{P}\left(X_{i: n}^{*}>t_{2}\right)} \frac{\sum_{k=1}^{n} s_{k} \mathbb{P}\left(X_{k: n}^{*}>t_{2}\right)}{\sum_{k=1}^{n} s_{k} \mathbb{P}\left(X_{k: n}^{*}>t_{1}\right)}
$$

and

$$
\frac{p_{j}\left(t_{1}\right)}{p_{j}\left(t_{2}\right)}=\frac{s_{j} \mathbb{P}\left(X_{j: n}^{*}>t_{1}\right)}{s_{j} \mathbb{P}\left(X_{j: n}^{*}>t_{2}\right)} \frac{\sum_{k=1}^{n} s_{k} \mathbb{P}\left(X_{k: n}^{*}>t_{2}\right)}{\sum_{k=1}^{n} s_{k} \mathbb{P}\left(X_{k: n}^{*}>t_{1}\right)}
$$


Hence,

$$
\frac{p_{i}\left(t_{1}\right)}{p_{i}\left(t_{2}\right)} \geq \frac{p_{j}\left(t_{1}\right)}{p_{j}\left(t_{2}\right)}
$$

if and only if

$$
\frac{\mathbb{P}\left(X_{i: n}^{*}>t_{1}\right)}{\mathbb{P}\left(X_{i: n}^{*}>t_{2}\right)} \geq \frac{\mathbb{P}\left(X_{j: n}^{*}>t_{1}\right)}{\mathbb{P}\left(X_{j: n}^{*}>t_{2}\right)}
$$

since $s_{i}, s_{j}>0$. This last expression holds whenever $X_{i: n}^{*} \leq_{\mathrm{hr}} X_{j: n}^{*}$ holds. But, under the PHR assumption, we know that $X_{i: n}^{*} \leq_{\operatorname{lr}} X_{j: n}^{*}$ holds. Also, it is well known that the likelihood ratio order implies the hazard rate order. Hence, $p_{i}\left(t_{1}\right) / p_{i}\left(t_{2}\right)$ is decreasing in $i$ (in their common support) and then $\boldsymbol{p}\left(t_{1}\right) \leq_{\operatorname{lr}} \boldsymbol{p}\left(t_{2}\right)$. The proof of the other case is similar.

Remark 2.1. Exploiting known results for orderings of sequential order statistics based on $F_{1}, \ldots, F_{n}$ (see Navarro and Burkschat (2011)), similar results can be obtained by replacing the PHR assumption with other conditions. For instance, if $h_{1}, \ldots, h_{n}$ denote the respective hazard rates of $F_{1}, \ldots, F_{n}$ and the ratios $h_{k} / k_{k+1}$ are nondecreasing functions for $k=1, \ldots, n-1$, then $X_{r: n}^{*} \leq \mathrm{hr} X_{r+1: n}^{*}$ holds for $r=1, \ldots, n-1$ and, therefore, the vector of coefficients (2.2) for the corresponding mixed system based on sequential order statistics also satisfies $\boldsymbol{p}\left(t_{1}\right) \leq \operatorname{lr} \boldsymbol{p}\left(t_{2}\right)$ for $0 \leq t_{1} \leq t_{2}$.

For systems with i.i.d. components, it has been shown in Navarro et al. (2008a) that

$$
\lim _{t \rightarrow \infty} \boldsymbol{p}(t)=(\underbrace{0, \ldots, 0}_{j-1 \text { times }}, 1, \underbrace{0, \ldots, 0}_{n-j \text { times }})
$$

holds for systems with a signature vector $s=\left(s_{1}, \ldots, s_{j}, 0, \ldots, 0\right)$ with $s_{j}>0$, that is, in the limit the complete probability mass is concentrated in only one entry of the vector. In the case of sequential order statistics, the limit behavior may be different, as we illustrate in Example 2.1 below. This example also proves that this property is not necessarily true for coherent systems based on exchangeable components.

Since we make use of the marginal distributions of generalized order statistics in this example, we recall here some particular results. If the parameters $\gamma_{1}, \ldots, \gamma_{n}$ are pairwise different, i.e. $\gamma_{i} \neq \gamma_{j}$ for $i \neq j$, then it is known (see Kamps and Cramer (2001)) that the distribution function of the $r$ th generalized order statistic and, if $F$ is absolutely continuous with density $f$, the corresponding density function are given by

$$
\begin{gathered}
F_{X_{*}^{(r)}}(t)=1-c_{r-1} \sum_{i=1}^{r} \frac{a_{i, r}}{\gamma_{i}}(1-F(t))^{\gamma_{i}}, \\
f_{X_{*}^{(r)}}(t)=c_{r-1} \sum_{i=1}^{r} a_{i, r}(1-F(t))^{\gamma_{i}-1} f(t),
\end{gathered}
$$

with the constants

$$
c_{r-1}=\prod_{j=1}^{r} \gamma_{j}, \quad a_{i, r}=\prod_{\substack{j=1 \\ j \neq i}}^{r} \frac{1}{\gamma_{j}-\gamma_{i}}, \quad 1 \leq i \leq r \leq n .
$$

The empty product $\prod_{\varnothing}$ is defined to be 1 . 
Example 2.1. Consider the system lifetime

$$
T=\max \left\{X_{1}^{*}, \min \left\{X_{2}^{*}, X_{3}^{*}\right\}\right\}
$$

with failure-dependent component lifetimes $X_{1}^{*}, X_{2}^{*}$, and $X_{3}^{*}$. The signature of this system is given by $s=\left(0, \frac{2}{3}, \frac{1}{3}\right)$ (see, e.g. Navarro and Rychlik (2007)). Let the lifetimes satisfy the PHR assumption (1.3) with $\alpha_{1}=1, \alpha_{2}=1.6$, and $\alpha_{3}=3.4$, and an underlying standard exponential distribution, i.e. $F(t)=1-\mathrm{e}^{-t}, t \geq 0$. Then, in particular, the corresponding parameters $\gamma_{1}$, $\gamma_{2}$, and $\gamma_{3}$ are strictly increasingly ordered:

$$
\gamma_{1}=3<\gamma_{2}=3.2<\gamma_{3}=3.4 \text {. }
$$

Because $s_{1}=0$, we obtain the following functions:

$$
\begin{gathered}
p_{1}(t)=0, \quad p_{2}(t)=\frac{2 \mathbb{P}\left(X_{2: 3}^{*}>t\right)}{2 \mathbb{P}\left(X_{2: 3}^{*}>t\right)+\mathbb{P}\left(X_{3: 3}^{*}>t\right)}, \\
p_{3}(t)=\frac{\mathbb{P}\left(X_{3: 3}^{*}>t\right)}{2 \mathbb{P}\left(X_{2: 3}^{*}>t\right)+\mathbb{P}\left(X_{3: 3}^{*}>t\right)} .
\end{gathered}
$$

By utilizing (2.6), the survival functions of the relevant sequential order statistics are given by

$$
\mathbb{P}\left(X_{2: 3}^{*}>t\right)=16 \mathrm{e}^{-3 t}-15 \mathrm{e}^{-3.2 t}, \quad \mathbb{P}\left(X_{3: 3}^{*}>t\right)=136 \mathrm{e}^{-3 t}-255 \mathrm{e}^{-3.2 t}+120 \mathrm{e}^{-3.4 t} .
$$

After substituting these expressions into (2.8), it can be shown that

$$
\lim _{t \rightarrow \infty} \boldsymbol{p}(t)=\left(0, \frac{4}{21}, \frac{17}{21}\right) \neq(0,0,1) .
$$

Analogously, it can be seen that

$$
\lim _{t \rightarrow 0+} \tilde{\boldsymbol{p}}(t)=(0,1,0)
$$

and

$$
\lim _{t \rightarrow \infty} \widetilde{\boldsymbol{p}}(t)=\left(0, \frac{2}{3}, \frac{1}{3}\right) .
$$

In fact, by making use of known limits of Meijer's $G$-function given in Cramer et al. (2004) and Bieniek (2007), the limits of the coefficients in Theorem 2.1 can be completely determined. The corresponding result is based on the next lemma. In order to simplify the presentation, we use the notation

$$
\gamma_{1: r}=\min \left\{\gamma_{1}, \ldots, \gamma_{r}\right\}, \quad 1 \leq r \leq n .
$$

Lemma 2.2. Let the PHR assumption (1.3) hold. If $1 \leq r<k \leq n$ then

$$
\lim _{t \rightarrow \infty} \frac{\mathbb{P}\left(X_{r: n}^{*}>t\right)}{\mathbb{P}\left(X_{k: n}^{*}>t\right)}=\prod_{l=r+1}^{k} \frac{\gamma_{l}-\gamma_{1: k}}{\gamma_{l}}
$$

and

$$
\lim _{t \rightarrow 0+} \frac{\mathbb{P}\left(X_{k: n}^{*} \leq t\right)}{\mathbb{P}\left(X_{r: n}^{*} \leq t\right)}=0 .
$$


Proof. Owing to the quantile transformation (2.5), it is sufficient to consider uniform generalized order statistics $U_{r: n}^{*}, r=1, \ldots, n$. From results given in Cramer and Kamps (2003), it follows that the marginal density function of the $r$ th uniform generalized order statistic with arbitrary model parameters $\gamma_{1}, \ldots, \gamma_{r}>0$ is given by

$$
f_{U_{r: n}^{*}}(t)=c_{r-1} G_{r}(t), \quad t \in(0,1),
$$

where $c_{r-1}$ is the constant given in (2.7) and

$$
G_{r}(t)=G_{r, r}^{r, 0}\left[\begin{array}{l|c}
1-t & \gamma_{1}, \ldots, \gamma_{r} \\
\gamma_{1}-1, \ldots, \gamma_{r}-1
\end{array}\right], \quad t \in(0,1),
$$

denotes a particular Meijer's $G$-function (for its definition, see, e.g. Luke (1969) or Mathai (1993)). For proving (2.9), we obtain, by utilizing l'Hôpital's rule and (2.11),

$$
\lim _{t \rightarrow 1-} \frac{\mathbb{P}\left(U_{r: n}^{*}>t\right)}{\mathbb{P}\left(U_{k: n}^{*}>t\right)}=\lim _{t \rightarrow 1-} \frac{c_{r-1} G_{r}(t)}{c_{k-1} G_{k}(t)} .
$$

By applying Lemma 3 of Bieniek (2007), it follows that

$$
\lim _{t \rightarrow 1-} \frac{G_{r}(t)}{G_{k}(t)}= \begin{cases}0, & r<\ell_{k}, \\ \prod_{l=r+1}^{k}\left(\gamma_{l}-\gamma_{\ell_{k}}\right), & r \geq \ell_{k},\end{cases}
$$

with $\ell_{k}=\max \left\{1 \leq j \leq k: \gamma_{j}=\gamma_{1: k}\right\}$. Since $\gamma_{\ell_{k}}=\gamma_{1: k}$, this proves the first assertion. Let us turn to the proof of (2.10). Arguing analogously to the above, we obtain

$$
\lim _{t \rightarrow 0+} \frac{\mathbb{P}\left(U_{k: n}^{*} \leq t\right)}{\mathbb{P}\left(U_{r: n}^{*} \leq t\right)}=\lim _{t \rightarrow 0+} \frac{c_{k-1} G_{k}(t)}{c_{r-1} G_{r}(t)} .
$$

It can be shown (see Equations (6) and (12) of Bieniek (2007), and also Cramer et al. (2004)) that

$$
\lim _{t \rightarrow 0+} \frac{G_{k}(t)}{G_{r}(t)}=0 .
$$

Hence, the second assertion is also proven.

Remark 2.2. If $\gamma_{i} \neq \gamma_{j}$ for $i \neq j$ then the proof of Lemma 2.2 can be directly obtained as follows. Let us consider the first limit. From (2.6), if $\gamma_{1: k}<\gamma_{1: r}$, we have

$$
\lim _{t \rightarrow \infty} \frac{\mathbb{P}\left(X_{r: n}^{*}>t\right)}{\mathbb{P}\left(X_{k: n}^{*}>t\right)}=0 .
$$

Analogously, if $\gamma_{1: k}=\gamma_{1: r}=\gamma_{i}$, we have

$$
\lim _{t \rightarrow \infty} \frac{\mathbb{P}\left(X_{r: n}^{*}>t\right)}{\mathbb{P}\left(X_{k: n}^{*}>t\right)}=\frac{c_{r-1} a_{i, r}}{c_{k-1} a_{i, k}} .
$$

Hence, (2.9) follows from (2.7). To show the second limit, consider

$$
\lim _{t \rightarrow 0+} \frac{\mathbb{P}\left(X_{r+1: n}^{*} \leq t\right)}{\mathbb{P}\left(X_{r: n}^{*} \leq t\right)}=\lim _{t \rightarrow 0+} \frac{c_{r} \sum_{i=1}^{r+1} a_{i, r+1}(1-t)^{\gamma_{i}-1}}{c_{r-1} \sum_{i=1}^{r} a_{i, r}(1-t)^{\gamma_{i}-1}}=\lim _{z \rightarrow 1-} \frac{\gamma_{r+1} \sum_{i=1}^{r+1} a_{i, r+1} z^{\gamma_{i}}}{\sum_{i=1}^{r} a_{i, r} z^{\gamma_{i}}} .
$$


Since $\sum_{i=1}^{r} a_{i, r} \gamma_{i}^{p-1}=0$ for $1 \leq p \leq r-1$ (see Balakrishnan et al. (2001, Remark 2.8)), we arrive at the following relation after invoking l'Hôpital $r-1$ times:

$$
\lim _{t \rightarrow 0+} \frac{\mathbb{P}\left(X_{r+1: n}^{*} \leq t\right)}{\mathbb{P}\left(X_{r: n}^{*} \leq t\right)}=\lim _{z \rightarrow 1-} \frac{\gamma_{r+1} \sum_{i=1}^{r+1} a_{i, r+1} \gamma_{i}^{r-1} z^{\gamma_{i}}}{\sum_{i=1}^{r} a_{i, r} \gamma_{i}^{r-1} z^{\gamma_{i}}} .
$$

Using the identity

$$
\sum_{i=1}^{r} a_{i, r} \gamma_{i}^{r-1}=(-1)^{r+1}
$$

(2.10) follows. The preceding identity can be shown by utilizing the approach via Lagrange interpolation polynomials in Balakrishnan et al. (2001) with the functional relation for $v_{r}(x)=$ $x^{r}$ evaluated at $x=0$.

From the definition of the coefficients (2.2) and (2.4), it is obvious that

$$
\lim _{t \rightarrow 0+} \boldsymbol{p}(t)=\boldsymbol{s}=\lim _{t \rightarrow \infty} \tilde{\boldsymbol{p}}(t) .
$$

In the following theorem the corresponding opposite limits are considered. The result for the coefficients in the inactivity time is the same as that given in Zhang (2010a) for i.i.d. component lifetimes. However, as we illustrated earlier, the limits for the residual lifetimes may be different from those obtained in Navarro et al. (2008a) for the i.i.d. case.

Theorem 2.3. Let $j \in\{1, \ldots, n\}$. Let $s=\left(s_{1}, \ldots, s_{n}\right)$ denote the signature of a mixed system with failure-dependent component lifetimes, and let $\boldsymbol{p}(t)$ and $\widetilde{\boldsymbol{p}}(t)$ denote the respective vectors of coefficients (2.2) and (2.4). Let the PHR assumption (1.3) hold.

(a) Let $s=\left(s_{1}, \ldots, s_{j}, 0, \ldots, 0\right)$ with $s_{j}>0$. If $i=\max \left\{1 \leq d \leq j: \gamma_{d}=\gamma_{1: j}\right\}$ then $\lim _{t \rightarrow \infty} p_{k}(t)=0$ for $1 \leq k \leq i-1$ and

$$
\lim _{t \rightarrow \infty} p_{k}(t)=s_{k}\left(\sum_{r=i}^{k-1} s_{r} \prod_{l=r+1}^{k} \frac{\gamma_{l}-\gamma_{1: k}}{\gamma_{l}}+s_{k}+\sum_{r=k+1}^{j} s_{r} \prod_{l=k+1}^{r} \frac{\gamma_{l}}{\gamma_{l}-\gamma_{1: r}}\right)^{-1}
$$

for $i \leq k \leq j$. In particular, if $\gamma_{1} \geq \cdots \geq \gamma_{j}$ then

$$
\lim _{t \rightarrow \infty} p(t)=(\underbrace{0, \ldots, 0}_{j-1 \text { times }}, 1, \underbrace{0, \ldots, 0}_{n-j \text { times }}) .
$$

(b) If $s=\left(0, \ldots, 0, s_{j}, \ldots, s_{n}\right)$ with $s_{j}>0$ then

$$
\lim _{t \rightarrow 0+} \tilde{\boldsymbol{p}}(t)=(\underbrace{0, \ldots, 0}_{j-1 \text { times }}, 1, \underbrace{0, \ldots, 0}_{n-j \text { times }}) .
$$

Proof. For proving (a), recall the representation given in Theorem 2.1(a):

$$
p_{k}(t)=\frac{s_{k} \mathbb{P}\left(X_{k: n}^{*}>t\right)}{\sum_{r=1}^{j} s_{r} \mathbb{P}\left(X_{r: n}^{*}>t\right)}, \quad t \geq 0 .
$$

Since $s_{k}=0$ for $k=j+1, \ldots, n$ by assumption, we obviously have

$$
p_{k}(t)=0, \quad k=j+1, \ldots, n .
$$


Now consider $k \in\{1, \ldots, j\}$. Because

$$
\lim _{t \rightarrow \infty} p_{k}(t)=s_{k} \lim _{t \rightarrow \infty}\left(\sum_{r=1}^{k-1} s_{r} \frac{\mathbb{P}\left(X_{r: n}^{*}>t\right)}{\mathbb{P}\left(X_{k: n}^{*}>t\right)}+s_{k}+\sum_{r=k+1}^{j} s_{r} \frac{\mathbb{P}\left(X_{r: n}^{*}>t\right)}{\mathbb{P}\left(X_{k: n}^{*}>t\right)}\right)^{-1},
$$

the assertion then follows by applying the first limit in Lemma 2.2. Assertion (b) follows similarly by applying the second limit.

Remark 2.3. Under the assumption that $\gamma_{1} \geq \cdots \geq \gamma_{j}$ given in Theorem 2.3, we obtain the same limits as in the i.i.d. case (in fact, the case of i.i.d. components is included in the result by choosing $\gamma_{k}=n-k+1,1 \leq k \leq n$ ). The ordering assumption on the $\gamma_{k}$ s has the following natural interpretation. Whereas the hazard rate of a component at a time $t$ between the $(k-1)$ th and $k$ th failure is given by $\alpha_{k} \lambda_{F}(t)$ (see (1.4)), the expression $\alpha_{k}(n-k+1) \lambda_{F}(t)=\gamma_{k} \lambda_{F}(t)$ can be interpreted as the hazard of the next failure among the $n-k+1$ active components at this time $t(1 \leq k \leq n)$. We would expect that the load on a component is nondecreasing after a failure, i.e. $\alpha_{1} \leq \cdots \leq \alpha_{n}$, but that the load on the system itself is nonincreasing, i.e. $\gamma_{1} \geq \cdots \geq \gamma_{n}$. Since the number of components is decreasing after each failure, the risk for the next failure among the still active components should decrease. If we assume (without loss of generality) that $\alpha_{1}=1$ then there are two extreme situations: (a) no additional load is imposed on each component after a failure $\left(\alpha_{1}=\cdots=\alpha_{n}=1\right)$ and the risk for the next failure among the active components is proportional to their number; (b) after a failure the initial load on the system is completely redistributed among the remaining components $\left(\gamma_{1}=\cdots=\gamma_{n}=n\right.$ and $\left.\alpha_{k}=n /(n-k+1), 1 \leq k \leq n\right)$, that is, the load on the system remains constant (see Balakrishnan et al. (2011, Example 1)).

\section{Mixture representations using additional information about a previous failure time}

In this section we derive representations of the residual lifetime and the inactivity time of a system given some information about times of failures. It is assumed that the $i$ th failure time of the components is known and that, at this time, the system is still active.

Theorem 3.1. Let $s=\left(s_{1}, \ldots, s_{n}\right)$ denote the signature of a mixed system with failuredependent component lifetimes $X_{1}^{*}, \ldots, X_{n}^{*}$ based on $F_{1}, \ldots, F_{n}$, and let $T$ denote the corresponding system lifetime. Let $1 \leq i \leq n-1$, and let $Y_{1: n-i}^{*}, \ldots, Y_{n-i: n-i}^{*}$ denote sequential order statistics based on $H_{1}, \ldots, H_{n-i}$ with

$$
H_{j}(z)=\frac{F_{i+j}(z)-F_{i+j}(y)}{1-F_{i+j}(y)}, \quad z \geq y, j=1, \ldots, n-i .
$$

(a) Let $x \geq 0$ and $0<y \leq t$. If $\left(s_{i+1}, \ldots, s_{n}\right) \neq(0, \ldots, 0)$ then

$$
\mathbb{P}\left(T-t>x \mid T>t, X_{i: n}^{*}=y\right)=\sum_{j=1}^{n-i} p_{j}(y, t) \mathbb{P}\left(Y_{j: n-i}^{*}-t>x \mid Y_{j: n-i}^{*}>t\right)
$$

with

$$
p_{j}(y, t)=\frac{s_{i+j} \mathbb{P}\left(Y_{j: n-i}^{*}>t\right)}{\sum_{k=1}^{n-i} s_{i+k} \mathbb{P}\left(Y_{k: n-i}^{*}>t\right)}, \quad j=1, \ldots, n-i .
$$


(b) Let $0 \leq x \leq t-y$ and $0<y<t$. If $\left(s_{i+1}, \ldots, s_{n}\right) \neq(0, \ldots, 0)$ then

$$
\mathbb{P}\left(t-T>x \mid y<T \leq t, X_{i: n}^{*}=y\right)=\sum_{j=1}^{n-i} \tilde{p}_{j}(y, t) \mathbb{P}\left(t-Y_{j: n-i}^{*}>x \mid Y_{j: n-i}^{*} \leq t\right)
$$

with

$$
\tilde{p}_{j}(y, t)=\frac{s_{i+j} \mathbb{P}\left(Y_{j: n-i}^{*} \leq t\right)}{\sum_{k=1}^{n-i} s_{i+k} \mathbb{P}\left(Y_{k: n-i}^{*} \leq t\right)}, \quad j=1, \ldots, n-i .
$$

Proof. We only prove (a), because (b) is shown similarly. Consider

$$
\mathbb{P}\left(T-t>x \mid T>t, X_{i: n}^{*}=y\right)=\sum_{j=1}^{n} \mathbb{P}\left(T-t>x, T=X_{j: n}^{*} \mid T>t, X_{i: n}^{*}=y\right) .
$$

Note that

$$
\begin{aligned}
& \mathbb{P}\left(T-t>x, T=X_{j: n}^{*} \mid T>t, X_{i: n}^{*}=y\right) \\
& \quad=\mathbb{P}\left(T=X_{j: n}^{*} \mid T>t, X_{i: n}^{*}=y\right) \mathbb{P}\left(T-t>x \mid T=X_{j: n}^{*}, T>t, X_{i: n}^{*}=y\right)
\end{aligned}
$$

if $\mathbb{P}\left(T=X_{j: n}^{*}\right)>0$; otherwise, the probability is equal to 0 . The first factor in (3.4) is given by

$$
\begin{aligned}
\mathbb{P}\left(T=X_{j: n}^{*} \mid T>t, X_{i: n}^{*}=y\right) & =\frac{\mathbb{P}\left(T=X_{j: n}^{*}, T>t \mid X_{i: n}^{*}=y\right)}{\mathbb{P}\left(T>t \mid X_{i: n}^{*}=y\right)} \\
& =\frac{\mathbb{P}\left(T=X_{j: n}^{*}, X_{j: n}^{*}>t \mid X_{i: n}^{*}=y\right)}{\mathbb{P}\left(T>t \mid X_{i: n}^{*}=y\right)} .
\end{aligned}
$$

Since the random variables $X_{1}^{*}, \ldots, X_{n}^{*}$ are exchangeable, the random variables $\mathbf{1}_{\left\{T=X_{j: n}^{*}\right\}}$ and $X_{1: n}^{*}, \ldots, X_{n: n}^{*}$ are independent. Here, $\mathbf{1}_{A}$ denotes the indicator function of the set $A$. Thus, by utilizing additionally that $X_{1: n}^{*} \leq \cdots \leq X_{n: n}^{*}$ almost surely, we obtain

$$
\begin{aligned}
\mathbb{P}(T & \left.=X_{j: n}^{*} \mid T>t, X_{i: n}^{*}=y\right) \\
& =\frac{\mathbb{P}\left(T=X_{j: n}^{*}\right) \mathbb{P}\left(X_{j: n}^{*}>t \mid X_{i: n}^{*}=y\right)}{\sum_{l=i+1}^{n} \mathbb{P}\left(T=X_{l: n}^{*}\right) \mathbb{P}\left(X_{l: n}^{*}>t \mid X_{i: n}^{*}=y\right)}, \quad j=i+1, \ldots, n,
\end{aligned}
$$

and this probability is equal to 0 for $j=1, \ldots, i$. Note that the denominator in (3.5) is positive by assumption. Furthermore, the second factor in (3.4) is given by

$$
\begin{aligned}
\mathbb{P}\left(T-t>x \mid T=X_{j: n}^{*}, T>t, X_{i: n}^{*}=y\right) & =\frac{\mathbb{P}\left(T-t>x, T=X_{j: n}^{*}, T>t \mid X_{i: n}^{*}=y\right)}{\mathbb{P}\left(T=X_{j: n}^{*}, T>t \mid X_{i: n}^{*}=y\right)} \\
& =\frac{\mathbb{P}\left(X_{j: n}^{*}>x+t, T=X_{j: n}^{*} \mid X_{i: n}^{*}=y\right)}{\mathbb{P}\left(X_{j: n}^{*}>t, T=X_{j: n}^{*} \mid X_{i: n}^{*}=y\right)} \\
& =\frac{\mathbb{P}\left(X_{j: n}^{*}>x+t \mid X_{i: n}^{*}=y\right)}{\mathbb{P}\left(X_{j: n}^{*}>t \mid X_{i: n}^{*}=y\right)},
\end{aligned}
$$


where in the last step we have again used the independence of $\mathbf{1}_{\left\{T=X_{j: n}^{*}\right\}}$ and $X_{1: n}^{*}, \ldots, X_{n: n}^{*}$. Furthermore, by exploiting the Markov property of sequential order statistics (see the transition probabilities (3.9) below), it can be shown that

$$
\mathbb{P}\left(X_{j: n}^{*}>t \mid X_{i: n}^{*}=y\right)=\mathbb{P}\left(Y_{j-i: n-i}^{*}>t\right), \quad t \geq y .
$$

This yields the assertion.

Remark 3.1. (a) Note that (3.1) and (3.3) coincide with the corresponding signature-based representations (2.1) and (2.3) of a mixed system consisting of $n-i$ components with the probability vector

$$
\boldsymbol{s}_{\mid i}=\left(\frac{s_{i+1}}{S_{i}}, \ldots, \frac{s_{n}}{S_{i}}\right), \quad S_{i}=s_{i+1}+\cdots+s_{n}(>0),
$$

and failure-dependent component lifetimes $Y_{1}^{*}, \ldots, Y_{n-i}^{*}$ based on $H_{1}, \ldots, H_{n-i}$ with support $[y, \infty)$. In particular, under the PHR assumption, properties analogous to the results given in Section 2 can be shown.

(b) It becomes obvious from the proof of Theorem 3.1 that, under the given assumptions,

$$
\begin{aligned}
& p_{j}(y, t)=\mathbb{P}\left(T=X_{j: n}^{*} \mid T>t, X_{i: n}^{*}=y\right), \\
& \tilde{p}_{j}(y, t)=\mathbb{P}\left(T=X_{j: n}^{*} \mid y<T \leq t, X_{i: n}^{*}=y\right)
\end{aligned}
$$

hold for $j=i+1, \ldots, n$. Therefore, the weights can be interpreted as the probabilities that the $j$ th failure leads to the system failure given the respective information on the system lifetime and the $i$ th failure time.

(c) The representations in Theorem 3.1 can be alternatively obtained by showing that

$$
\mathbb{P}\left(T \in B \mid X_{i: n}^{*}=y\right)=\sum_{j=1}^{n} s_{j} \mathbb{P}\left(X_{j: n}^{*} \in B \mid X_{i: n}^{*}=y\right)
$$

for Borel sets $B \subset \mathbb{R}$ at first and then considering appropriate ratios of these expressions. In fact, this derivation relies on the same arguments as those given in the proof of Theorem 3.1.

(d) Additional conditioning under earlier failure times $X_{i-1: n}^{*}, \ldots, X_{1: n}^{*}$ also leads to the expressions given in Theorem 3.1, since sequential order statistics possess the Markov property.

Example 3.1. Let the lifetime of a system be described by

$$
T=\min \left\{X_{1}^{*}, \max \left\{X_{2}^{*}, X_{3}^{*}, X_{4}^{*}\right\}\right\}
$$

with $X_{1}^{*}, \ldots, X_{4}^{*}$ denoting failure-dependent lifetimes of four components. Let the PHR assumption (1.3) be fulfilled with the parameters $\alpha_{1}=1, \alpha_{2}=1.2, \alpha_{3}=1.4$, and $\alpha_{4}=1.6$. Moreover, assume here that the time axis is chosen in such a way that the underlying distribution function $F$ is given by the standard exponential distribution $F(t)=1-\mathrm{e}^{-t}, t \geq 0$. Note that the signature of the given system is the vector $s=\left(\frac{1}{4}, \frac{1}{4}, \frac{1}{2}, 0\right)$. Suppose that the first failure of a component is recorded at time $y=0.6$ and that the system is still active after that failure. In particular, only one of the components belonging to the lifetimes $X_{2}^{*}, X_{3}^{*}$, and $X_{4}^{*}$ must have failed. Furthermore, an inspection at time $t=1.5$ reveals that the system is not 
operating anymore. If no data is available about further failures of components between the times $y$ and $t$, it is of interest to study the distribution of the inactivity time of the system given the information provided so far. Theorem 3.1 yields

$$
\begin{aligned}
\mathbb{P}(t- & \left.T>x \mid y<T \leq t, X_{1: 3}^{*}=y\right) \\
& =\sum_{j=1}^{3} \tilde{p}_{j}(y, t) \mathbb{P}\left(t-Y_{j: 3}^{*}>x \mid Y_{j: 3}^{*} \leq t\right), \quad 0 \leq x \leq 0.9,
\end{aligned}
$$

with the weights (because $s_{4}=0$ )

$$
\begin{gathered}
\tilde{p}_{1}(y, t)=\frac{\mathbb{P}\left(Y_{1: 3}^{*} \leq t\right)}{\mathbb{P}\left(Y_{1: 3}^{*} \leq t\right)+2 \mathbb{P}\left(Y_{2: 3}^{*} \leq t\right)}, \\
\tilde{p}_{2}(y, t)=\frac{2 \mathbb{P}\left(Y_{2: 3}^{*} \leq t\right)}{\mathbb{P}\left(Y_{1: 3}^{*} \leq t\right)+2 \mathbb{P}\left(Y_{2: 3}^{*} \leq t\right)}, \quad \tilde{p}_{3}(y, t)=0,
\end{gathered}
$$

where $Y_{1: 3}^{*}, Y_{2: 3}^{*}$, and $Y_{3: 3}^{*}$ denote sequential order statistics based on the new parameters $\widetilde{\alpha}_{1}=$ $1.2, \widetilde{\alpha}_{2}=1.4$, and $\widetilde{\alpha}_{3}=1.6$, and with underlying (truncated) distribution function $H(z)=$ $1-\mathrm{e}^{-(z-y)}, z \geq y$. Observe that, in fact, probability (3.6) does not depend on the last parameter $\alpha_{4}=\tilde{\alpha}_{3}$. By plugging in the known values $y=0.6$ and $t=1.5$, and utilizing (2.6), it can be shown via numerical evaluation that

$$
\tilde{p}_{1}(0.6,1.5)=1-\tilde{p}_{2}(0.6,1.5) \approx 0.38267 .
$$

Therefore, it is more likely that the third failure among the components led to the system failure (see Remark 3.1(b)). Moreover, since we obtain

$$
\mathbb{E}\left(1.5-T \mid 0.6<T \leq 1.5, X_{1: 3}^{*}=0.6\right) \approx 0.53597,
$$

it is to be expected that the system failed some time before $t=1.5$.

As a corollary, we obtain the following result given in Navarro and Burkschat (2011). For completeness, we give the new proof.

Corollary 3.1. Let $s=\left(s_{1}, \ldots, s_{n}\right)$ denote the signature of a mixed system with failuredependent component lifetimes $X_{1}^{*}, \ldots, X_{n}^{*}$ based on $F_{1}, \ldots, F_{n}$, and let $T$ denote the corresponding system lifetime. Let $1 \leq i \leq n-1$. Then the distribution of $\left(T-t \mid X_{i: n}^{*}=\right.$ $t<T$ ) coincides with the distribution of the lifetime of a mixed system with $n-i$ components and signature

$$
\boldsymbol{s}_{\mid i}=\left(\frac{s_{i+1}}{S_{i}}, \ldots, \frac{s_{n}}{S_{i}}\right)
$$

(with $S_{i}=s_{i+1}+\cdots+s_{n}>0$ ) based on the sequential order statistics with underlying distributions $G_{1}, \ldots, G_{n-i}$ with

$$
G_{j}(x)=\frac{F_{i+j}(x+t)-F_{i+j}(t)}{1-F_{i+j}(t)}, \quad x \geq 0, j=1, \ldots, n-i .
$$

Proof. We get the result by choosing $t=y$ in Theorem 3.1(a). This is seen as follows. At first, because $Y_{j: n-i}^{*}>y$ holds almost surely for $j=1, \ldots, n-i$, it follows that, for the vector of coefficients in (3.2), $\boldsymbol{p}(y, y)=\boldsymbol{s}_{\mid i}$. Moreover, if $t=y$ then

$$
\mathbb{P}\left(Y_{j: n-i}^{*}-t>x \mid Y_{j: n-i}^{*}>t\right)=\mathbb{P}\left(Y_{j: n-i}^{*}-y>x\right)
$$


and the joint distribution of $Y_{1: n-i}^{*}-y, \ldots, Y_{n-i: n-i}^{*}-y$ with underlying $H_{1}, \ldots, H_{n-i}$ coincides with the distribution of sequential order statistics $Z_{1: n-i}^{*}, \ldots, Z_{n-i: n-i}^{*}$ based on $G_{1}, \ldots, G_{n-i}$ (the latter relation is seen from the corresponding transition probabilities of the sequential order statistics; cf. (3.9) below).

Finally, we establish a connection to the dynamic signature representation given in Samaniego et al. (2009) for systems with i.i.d. component lifetimes $X_{1}, \ldots, X_{n}$ based on $F$. From the results in this paper, it follows that (for $1 \leq i \leq n-1$ )

$$
\mathbb{P}\left(T-t>x \mid T>t, X_{i: n} \leq t<X_{i+1: n}\right)=\sum_{j=1}^{n-i} \frac{s_{i+j}}{S_{i}} \mathbb{P}\left(Z_{j: n-i}>x\right)
$$

with the constant $S_{i}$ from Corollary 3.1 and order statistics $Z_{1: n-i}, \ldots, Z_{n-i: n-i}$ from i.i.d. random variables with distribution function

$$
G(x)=\frac{F(x+t)-F(t)}{1-F(t)}, \quad x \geq 0 .
$$

In fact, the preceding corollary can be regarded as an extension of representation (3.7) to sequential order statistics. This follows from the distribution theoretical relation

$$
\left(X_{i+1: n}^{*}, \ldots, X_{n: n}^{*} \mid X_{i: n}^{*}=y\right) \sim\left(X_{i+1: n}^{*}, \ldots, X_{n: n}^{*} \mid X_{i: n}^{*} \leq y<X_{i+1: n}^{*}\right)
$$

for sequential order statistics. Related recent results in settings of generalized order statistics can be found in Xie and $\mathrm{Hu}$ (2008), Balakrishnan et al. (2009), Burkschat and Lenz (2009), and Hashemi et al. (2010). Here we give some arguments for the univariate relation, i.e. for $1 \leq i<j \leq n$, the equality

$$
\mathbb{P}\left(X_{j: n}^{*}>t \mid X_{i: n}^{*} \leq y<X_{i+1: n}^{*}\right)=\mathbb{P}\left(X_{j: n}^{*}>t \mid X_{i: n}^{*}=y\right)
$$

holds for $\mathbb{P}_{X_{i: n}^{*}}$-almost all $y \geq 0$. The general relation in the multivariate case follows analogously. At first, let $j=i+1$. It is well known that (cf. Kamps (1995a, p. 29))

$$
\mathbb{P}\left(X_{i+1: n}^{*}>t \mid X_{i: n}^{*}=y\right)= \begin{cases}\left(\frac{1-F_{i+1}(t)}{1-F_{i+1}(y)}\right)^{n-i}, & t \geq y, \\ 1, & t<y,\end{cases}
$$

for $\mathbb{P}_{X_{i: n}^{*}}$-almost all $y \geq 0$. In particular, we obtain

$$
\begin{aligned}
& \mathbb{P}\left(X_{i+1: n}^{*}>t, X_{i: n}^{*} \leq y<X_{i+1: n}^{*}\right) \\
& \quad=\int_{0}^{y}\left(\frac{1-F_{i+1}(\max \{y, t\})}{1-F_{i+1}(z)}\right)^{n-i} \mathrm{~d} \mathbb{P}_{X_{i: n}^{*}}(z), \quad t \geq 0 .
\end{aligned}
$$

Dividing by

$$
\mathbb{P}\left(X_{i: n}^{*} \leq y<X_{i+1: n}^{*}\right)=\int_{0}^{y}\left(\frac{1-F_{i+1}(y)}{1-F_{i+1}(z)}\right)^{n-i} \mathrm{~d} \mathbb{P}_{X_{i: n}^{*}}(z)
$$

and exploiting (3.9) yields relation (3.8) for $j=i+1$. Now, let $n \geq j>i+1$. Then

$$
\begin{aligned}
& \mathbb{P}\left(X_{j: n}^{*}>t \mid X_{i: n}^{*} \leq y<X_{i+1: n}^{*}\right) \\
& \quad=\int_{y}^{\infty} \mathbb{P}\left(X_{j: n}^{*}>t \mid X_{i+1: n}^{*}=z, X_{i: n}^{*} \leq y<X_{i+1: n}^{*}\right) \mathrm{dP}_{X_{i+1: n}^{*} \mid X_{i: n}^{*} \leq y<X_{i+1: n}^{*}(z) .}
\end{aligned}
$$


Since sequential order statistics have the Markov property, the past and the future are conditionally independent given the present. Therefore, for $z>y$,

$$
\begin{aligned}
\mathbb{P}\left(X_{j: n}^{*}>t \mid X_{i+1: n}^{*}=z, X_{i: n}^{*} \leq y<X_{i+1: n}^{*}\right) & =\frac{\mathbb{P}\left(X_{j: n}^{*}>t, X_{i: n}^{*} \leq y \mid X_{i+1: n}^{*}=z\right)}{\mathbb{P}\left(X_{i: n}^{*} \leq y \mid X_{i+1: n}^{*}=z\right)} \\
& =\mathbb{P}\left(X_{j: n}^{*}>t \mid X_{i+1: n}^{*}=z\right) \\
& =\mathbb{P}\left(X_{j: n}^{*}>t \mid X_{i+1: n}^{*}=z, X_{i: n}^{*}=y\right),
\end{aligned}
$$

where in the last step we have again used the Markov property. By utilizing the preceding relation and the previously proven fact that (3.8) holds for $j=i+1$, we obtain, from (3.10),

$$
\begin{aligned}
\mathbb{P}\left(X_{j: n}^{*}>t \mid X_{i: n}^{*} \leq y<X_{i+1: n}^{*}\right) & \\
\quad= & \int_{y}^{\infty} \mathbb{P}\left(X_{j: n}^{*}>t \mid X_{i+1: n}^{*}=z, X_{i: n}^{*}=y\right) d \mathbb{P}_{X_{i+1: n}^{*} \mid X_{i: n}^{*}=y}(z) \\
& =\mathbb{P}\left(X_{j: n}^{*}>t \mid X_{i: n}^{*}=y\right)
\end{aligned}
$$

for $\mathbb{P}_{X_{i: n}^{*}}$-almost all $y \geq 0$.

\section{Acknowledgements}

We thank an anonymous referee for several helpful comments. JN was partially supported by Ministerio de Ciencia y Tecnología and Fundación Séneca, under grants MTM2009-08311 and $08627 / \mathrm{PI} / 08$.

\section{References}

Balakrishnan, N., Beutner, E. AND Kamps, U. (2011). Modeling parameters of a load-sharing system through link functions in sequential order statistics models and associated inference. IEEE Trans. Reliab. 60, 605-611.

Balakrishnan, N., Cramer, E. AND KAMPS, U. (2001). Bounds for means and variances of progressive type II censored order statistics. Statist. Prob. Lett. 54, 301-315.

Balakrishnan, N., Kamps, U. And Kateri, M. (2009). Minimal repair under a step-stress test. Statist. Prob. Lett. 79, $1548-1558$.

Barlow, R. E. and Proschan, F. (1981). Statistical Theory of Reliability and Life Testing. To Begin With, Silver Spring, MD.

BIENIEK, M. (2007). Variation diminishing property of densities of uniform generalized order statistics. Metrika $\mathbf{6 5}$, 297-309.

Burkschat, M. (2009). Systems with failure-dependent lifetimes of components. J. Appl. Prob. 46, 1052-1072.

Burkschat, M. AND Lenz, B. (2009). Marginal distributions of the counting process associated with generalized order statistics. Commun. Statist. Theory Methods 38, 2089-2106.

Burkschat, M. and Navarro, J. (2011). Aging properties of sequential order statistics. Prob. Eng. Inf. Sci. 25, 449-467.

Cramer, E. (2006). Sequential order statistics. In Encyclopedia of Statistical Sciences, Vol. 12, 2nd edn, eds S. Kotz et al., John Wiley, Hoboken, pp. 7629-7634.

Cramer, E. AND Kamps, U. (2001). Sequential $k$-out-of- $n$ systems. In Advances in Reliability (Handbook Statist. 20), eds N. Balakrishnan and C. R. Rao, North-Holland, Amsterdam, pp. 301-372.

Cramer, E. AND Kamps, U. (2003). Marginal distributions of sequential and generalized order statistics. Metrika 58, 293-310.

Cramer, E., Kamps, U. and RaqAB, M. Z. (2003). Characterizations of exponential distributions by spacings of generalized order statistics. Appl. Math. 30, 257-265.

Cramer, E., Kamps, U. AND Rychlik, T. (2004). Unimodality of uniform generalized order statistics, with applications to mean bounds. Ann. Inst. Statist. Math. 56, 183-192.

Hashemi, M., TAVAngar, M. AND Asadi, M. (2010). Some properties of the residual lifetime of progressively type-II right censored order statistics. Statist. Prob. Lett. 80, 848-859. 
Hu, T. And Zhuang, W. (2005). A note on stochastic comparisons of generalized order statistics. Statist. Prob. Lett. 72, $163-170$.

KAMPS, U. (1995a). A Concept of Generalized Order Statistics. Teubner, Stuttgart.

KAMPS, U. (1995b). A concept of generalized order statistics. J. Statist. Planning Inf. 48, 1-23.

KAMPS, U. AND CRAMER, E. (2001). On distributions of generalized order statistics. Statistics 35, 269-280.

Luke, Y. L. (1969). The Special Functions and Their Approximations, Vol. I. Academic Press, New York.

Mathai, A. M. (1993). A Handbook of Generalized Special Functions for Statistical and Physical Sciences. Clarendon Press, Oxford.

Navarro, J. And Burkschat, M. (2011). Coherent systems based on sequential order statistics. Naval Res. Logistics 58, $123-135$.

NAVARro, J. AND Rychlik, T. (2007). Reliability and expectation bounds for coherent systems with exchangeable components. J. Multivariate Anal. 98, 102-113.

Navarro, J. and Shaked, M. (2006). Hazard rate ordering of order statistics and systems. J. Appl. Prob. 43, $391-408$.

Navarro, J., Balakrishnan, N. And Samaniego, F. J. (2008a). Mixture representations of residual lifetimes of used systems. J. Appl. Prob. 45, 1097-1112.

Navarro, J., Samaniego, F. J., Balakrishnan, N. and Bhattacharya, D. (2008b). On the application and extension of system signatures in engineering reliability. Naval Res. Logistics 55, 313-327.

Samaniego, F. J. (1985). On closure of the IFR class under formation of coherent systems. IEEE Trans. Reliab. 34, 69-72.

Samaniego, F. J. (2007). System Signatures and Their Applications in Engineering Reliability. Springer, New York.

Samaniego, F. J., Balakrishnan, N. And Navarro, J. (2009). Dynamic signatures and their use in comparing the reliability of new and used systems. Naval Res. Logistics 56, 577-591.

Shaked, M. And Shanthikumar, J. G. (2007). Stochastic Orders. Springer, New York.

Xie, H. And Hu, T. (2008). Conditional ordering of generalized order statistics revisited. Prob. Eng. Inf. Sci. 22, $333-346$.

ZHANG, Z. (2010a). Mixture representations of inactivity times of conditional coherent systems and their applications. J. Appl. Prob. 47, 876-885.

ZHANG, Z. (2010b). Ordering conditional general coherent systems with exchangeable components. J. Statist. Planning Inf. 140, 454-460. 